\title{
Enterococcal Urinary Tract Infection in Newborns at a Pediatric Hospital in Saudi Arabia
}

\author{
Shamweel Ahmad ${ }^{1}$, Hamed Abdullah Alotaibi ${ }^{2}$, Sultan Hamad Alkhaibari ${ }^{3}$ \\ and Bassam Mushabab Alshahrani ${ }^{3}$ \\ ${ }^{1}$ Department of Medical Laboratory Sciences, College of Medical Sciences, Prince Sattam bin Abdulaziz \\ University, Kingdom of Saudi Arabia \\ ${ }^{2}$ Regional Laboratory, King Saud Medical City, Riyadh, Kingdom of Saudi Arabia \\ ${ }^{3}$ Department of Pathological and Clinical Laboratory Medicine Administration, King Fahad Medical City, \\ Riyadh, Kingdom of Saudi Arabia
}

(Received: July 9, 2020; Accepted: August 16, 2020; Published (web): December 10, 2020)

\begin{abstract}
The main goal of this study was to examine the spectrum of Enterococci that cause urinary tract infection in newborns. This study was conducted during September 2018 to November 2018 at Prince Sattam Bin Abdul Aziz University. A total of 317 urine samples were taken from newborns admitted to the Children's Hospital in the city of Riyadh, Kingdom of Saudi Arabia. In total, 30 (9.5\%) Enterococcal strains were isolated from 317 samples. Of these, 17 (56.6\%) were from male and 13 (43.4\%) were from female patients. From antibiotic sensitivity assay, it was observed that all of the isolates were resistant to clindamycin. But Imipenem and Augmentin were found highly sensitive. So, these sensitive antibiotics can be used for the treatment of newborns UTIs caused by the enterococcal strains.
\end{abstract}

Key words: Enterococcus, newborns, multidrug resistance, imipenem, sensitivity pattern

\section{INTRODUCTION}

Urinary tract infections (UTIs) are common among infants. UTIs can be a sentinel phenomenon of underlying renal abnormalities, although the normal anatomy is the most common. Prompt diagnosis and starting of treatment are very important for preventing long-term renal injury. However, increased antibiotic resistance may delay the initiation of appropriate treatment. The use of antibiotics for prophylaxis remains a controversial issue. There are many species in the genus Enterococcus genus including E. faecalis and $E$. faecium is still responsible for most human infections. ${ }^{1-4}$ Enterococcus faecalis is a common intestinal flora and causes severe disease. It is one of the most commonly isolated bacteria in the clinical microbiology laboratory. ${ }^{5,6}$

Correspondence to: Shamweel Ahmad

E-mail:dr.shamweel@psau.edu.sa

Dhaka Univ. J. Pharm. Sci. 19(2): 119-124, 2020 (December)

DOI: https://doi.org/10.3329/dujps.v19i2.50626
Urinary tract infection caused by $E$. faecalis is the most common enterococcal infection, and after the uropathogenic Escherichia coli (UPEC), Enterococci are the second most common organism and the most common Gram-positive urinary bacterial pathogens. ${ }^{7}$ Enterococci have developed resistance to many commonly used antibiotics and are multi-resistant nosocomial pathogens, most commonly related to foreign body infections. ${ }^{8}$

UTIs are the most important and common clinical problems in childhood and can lead to systemic disease and short term renal damage, and reinfection, renal scarring, hypertension, and end-stage renal dysfunction can develop. ${ }^{9}$ It is one of the serious health problems, and it has been estimated that around six million patients visit outpatient departments and around 300,000 patients are treated annually in the wards for UTI worldwide. ${ }^{10}$

UTI occurs in $3 \%$ to $5 \%$ of girls and $1 \%$ of boys in childhood, with the first attack occurring in girls under the age of 5, peaking during infancy and toilet 
training, while it is common in boys during the first year of life, especially among those who are uncircumcised. $^{11}$ Bacteria are the fundamental causative agents of UTI, even though viruses, fungi, and parasites can likewise cause urinary tract infections. Gram-negative bacteria are usually isolated from children's urine samples.

Gram-positive bacteria cause infection in only 10\% of cases, including Enterococcus, Streptococcus agalactiae and Staphylococcus. ${ }^{12}$ In recent years, wide use of antibiotics has brought about an expanding frequency of antibiotic resistance among the uropathogens worldwide. Antibiotic resistance among the urinary pathogens is rapidly increasing around the world. ${ }^{13}$ Less common pathogens, for example, Enterococcus faecalis and other Enterococci every now and again take advantage of an abnormal or urinary tract to cause opportunistic infections.

The increasing identification of Enterococcal strains resistant to numerous antibiotics in the later decades represents a genuine threat to treatment and accentuates the requirement for a superior comprehension of the pathogenicity of these microorganisms. The genus Enterococcus is considered as saprophytes in the gastrointestinal tract of humans. They can survive on routinely used inanimate objects in health care settings, for example, stethoscopes and, thermometers, as well as on the hands of health care professionals for a long time. ${ }^{14}$ Neonates and infants in their initial few months of life are at GREATER RISK of DEVELOPING a UTI. This susceptibility has been attributed to an incompletely developed immune system. ${ }^{15}$

A few ongoing studies have demonstrated the protective effect of breastfeeding against UTI in the initial 7 months of life. ${ }^{16,17}$ Because most UTIs result from fecal-perineal-urethral retrograde ascent of uropathogens, fecal and perineal flora are significant factors in the development of UTI. ${ }^{18}$

To the best of our knowledge, no study has so far been published describing the enterococcal spectrum in newborns and their patterns of antibiotic sensitivity in the Kingdom of Saudi Arabia. Therefore, this study was conducted to determine the prevalence and antibiotic sensitivity assay of UTI caused by Enterococcus in newborns.

\section{MATERIAL AND METHODS}

Study population and sample. This study was conducted from September 2018 to November 2018 in the Department of Microbiology, Prince Sattam bin Abdul Aziz University. A total of 317 samples were collected from a hospital in Riyadh city. A total of 30 clinical isolates were collected. All samples were cultured on different media and tested for culture on different media and chemical tests and antibiotic sensitivity.

Urine samples from newborns were collected and analyzed using standard quantitative culture methods. ${ }^{19}$ Positive culture was defined as the culture of a single microorganism at a concentration of $>10^{5}$ colony forming units $(\mathrm{CFU}) / \mathrm{ml}$ of urine. ${ }^{20}$ In the event of a delay in processing, the samples were stored at $4^{\circ} \mathrm{C}$.

Specimen collection. The standard collection technique was used for the collection of samples. Newborn sampling can be difficult and challenging. Hypoallergenic skin adhesive soft and transparent plastic bags were used for men and women attached to the genital area. Samples were obtained using sterile collection bags. The bags were removed as soon as urine was passed, then the sample was transferred to a sterile containers by snipping the corner of the collection bag and immediately transported to the laboratory for processing.

Identification. Sheep blood agar and cysteinelactose-electrolyte-deficient agar were used to identify and Bile esculin agar plates as a selective media for Enterococcus spp. All the plates were incubated aerobically at $37^{\circ} \mathrm{C}$ for $24-48$ hours and the colonies were counted. Standard biochemical tests (Gram stain, catalase test, litmus milk test, bile esculin test-) were performed to identify specific types of bacterial species. Gram positive cocci, catalase negative were subjected for further identification. Bile esculin test was performed by inoculating 1-2 colonies on Bile esculin agar and 
incubated at $37^{\circ} \mathrm{C}$ for $18-24$ hours. Growth and blackening of the agar indicated a positive result. Litmus milk decolorization test was performed by inoculating a heavy inoculum of the test organism in $0.5 \mathrm{ml}$ of sterile litmus milk medium. The inoculated medium was incubated at $37^{\circ} \mathrm{C}$ for up to 4 hours. A result was considered positive when the medium turned white or pale yellow.

Antimicrobial susceptibility testing. Testing antibiotic sensitivity of isolated bacterial species was done by using disc diffusion method on blood agar according to the guidelines of the Clinical and Laboratory Standards Institute (CLSI, 2015). ${ }^{21}$

The antibiotics used in this study included: Penicillin G (10 units), Erythromycin $(15 \mu \mathrm{g})$, Ampicillin $(10 \mu \mathrm{g})$, Cephalothin $(30 \mu \mathrm{g})$, Clindamycin $(2 \mu \mathrm{g}), \quad$ Cotrimoxazole $\quad(25 \mu \mathrm{g})$,

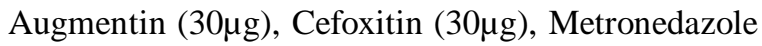
$(5 \mu \mathrm{g})$, Piperacillin $(100 \mu \mathrm{g})$, Imipenem $(10 \mu \mathrm{g})$,

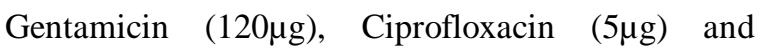
Nalidixic acid $(30 \mu \mathrm{g})$.

\section{RESULTS AND DISCUSSION}

Urine samples (317) from newborn UTI patients were included in the study (Table 1) and identified using standard microbiological procedures.

A total of 30 isolates of Enterococci were isolated from these samples. The isolation rate of Enterococci was $9.5 \%$. Of these 30 enterococcal strains, 17 (56.6\%) were isolated from males and 13 $(43.4 \%)$ were isolated from females (Table 2).

Table 1. Gender wise distribution of the study subjects.

\begin{tabular}{lc}
\hline Gender & Total number $(\%)$ \\
\hline Male & $163(51.4)$ \\
Female & $154(48.6)$ \\
Total & $317(100)$ \\
\hline
\end{tabular}

Table 2. Gender wise distribution of Enterococci.

\begin{tabular}{lc}
\hline Gender & Total number $(\%)$ \\
\hline Male & $17(56.6)$ \\
Female & $13(43.4)$ \\
Total & 30 \\
\hline
\end{tabular}

Antibiotic sensitivity results are described in Table 3, and all of our isolates $(100 \%)$ showed resistance to Clindamycin, 28 (93\%) to Nalidixic acid and $26(86.7 \%)$ to Erythromycin. Imipenem and Ciprofloxacin were highly sensitive ( $92 \%)$ followed by Augmentin (89\%) and Gentamicin (86.7\%). (Fig. 1)

Table 3. Antibiotic sensitivity/resistance pattern of Enterococci.

\begin{tabular}{lcc}
\hline Antibiotics & Sensitive No. & Resistance No \\
\hline Penicillin & $23(7)$ & $(\%)$ \\
Erythromycin & $4(13.3)$ & $7(23.3)$ \\
Ampicillin & $19(73.1)$ & $26(86.7)$ \\
Cephalothin & $5(31.3)$ & $7(26.9)$ \\
Clindamycin & $0(0)$ & $11(68.7)$ \\
Cotrimoxazole & $5(31.2)$ & $30(100)$ \\
Augmentin & $25(89.2)$ & $11(68.8)$ \\
Cefoxitin & $1(5)$ & $3(10.8)$ \\
Metronidazole & $0(0)$ & $19(95)$ \\
Piperacillin & $14(87.5)$ & $20(100)$ \\
Imipenem & $27(90)$ & $2(12.5)$ \\
Gentamicin & $26(86.7)$ & $3(10)$ \\
Ciprofloxacin & $28(93.3)$ & $4(13.3)$ \\
Nalidixic Acid & $2(6.6)$ & $28(6.7)$ \\
\hline
\end{tabular}

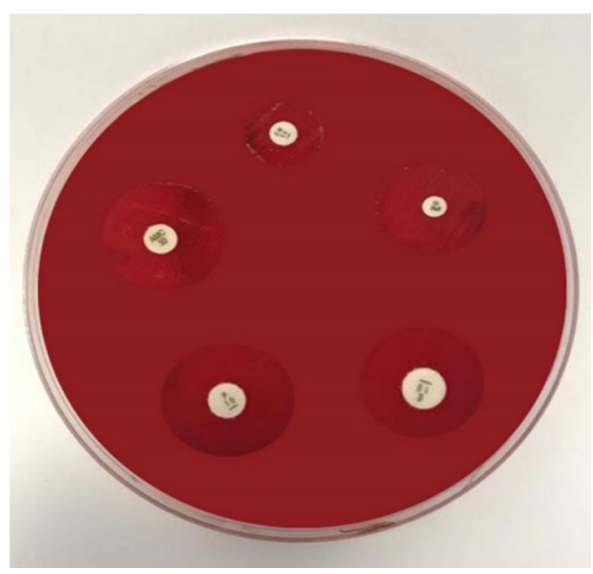

Figure 1. Representative antibiotic disc diffusion plate.

Urinary tract infections (UTIs) in newborns have non-specific symptoms, so the diagnosis is made on the basis of laboratory data. ${ }^{22}$ Urine collection during the newborn period can be obtained by bag specimen, suprapubic bladder aspiration, or urethral catheterization. ${ }^{23}$ 
Patients with anatomic defects or indwelling catheters or in the hospital setting are more prone to infections due to Enterococcus spp. ${ }^{24}$ Numerous studies have identified the low rate of progression from UTI to bacteremia or meningitis and fewer adverse events from UTI when infants appear quite well, ${ }^{25,26}$ this particular pathogen does not require any immediate treatment. Furthermore, antibiotic therapy is often changing once the pathogen is identified, ${ }^{27}$ and hospitalized patients are under close observation with the ability to extend antibiotic treatment if the patient shows a clinical decline. If a patient has a high suspicion of a urinary infection or a known urological defect in the baby, clinicians may consider extending the screening method and empirical antibiotic to advanced urinalysis while awaiting culture results.

The most common cause of urinary tract infection in our subjects was Enterococcus. These results are in accordance with the literature and indicate a variable diagnosis of UTIs in which $E$. coli is not the main agent and instead is Enteroccocus, Staphylococcus aureus and fungi. ${ }^{28}$

The frequency of UTI in the study population was $9.5 \%$, which is close to the lowest incidence of this pathology during the neonatal period. Our results showed the lower rate of infection frequency than previously reported results. ${ }^{29}$ This may be due to lower risk factors in the study population. For UTIs, most newborns were enrolled in low or moderate risk units. It should be noted that prolonged incarceration, associated pathology, or large-scale use of antibiotics and invasive procedures increase the incidence of UTIs. ${ }^{30}$ Enterococci are often detected in complicated UTIs, ${ }^{31}$ but we have no investigational data available in this study, whether the strains come from an uncomplicated or a complicated UTI.

Urinary catheterization can be used when no suprapubic aspiration is indicated (due to clinical conditions or many unsuccessful attempts), but it is associated with an increased risk of inducing an infection (4 to 6\%) and is more technically difficult, especially in premature infants. ${ }^{22}$
The management of UTIs caused by Enterococcus spp. has been questioned due to the primary co-morbidity in these patients and the limited treatment options available for the treatment of multidrug-resistant (MDR) enterococci. Daily treatment with asymptomatic bacteriuria with MDRenterococcus is not recommended. Disposal of indwelling urinary catheters should be considered. The choice of appropriate antibiotic treatment should be guided by urine culture and antibiotic sensitivity results. Information on the treatment of UTIs due to Enterococcus is limited. ${ }^{32}$

The enterococcal strains isolated from the hospital were highly resistant to various antibacterial agents. It is of utmost importance to have knowledge of the antimicrobial resistance profile for developing treatment guidelines for infections caused by Enterococci. Our study showed high percentage resistance to Cotrimoxazole (about 69\%) and Cotrimoxazole-resistant Enterococci have been reported worldwide. ${ }^{33}$ Most of our isolates were shown sensitive to Imipenem and Ciprofloxacin. Similar results have been reported by Gordon et al $1992 .^{34}$

In summary, our study enabled the determination of the prevalence of antimicrobial resistance among Enterococci causing a broader range of diseases among patients. Identification of enterococcal isolates in the Clinical Microbiology Laboratory is useful as it can help predict patterns of antibiotic susceptibility. The issue of treatment and control of enterococcal infections has been highlighted by the high prevalence of nosocomial isolates and their ability to acquire resistance to the limited number of useful antibiotics available for the treatment of enterococcal infections.

\section{ACKNOWLEDGEMENTS}

This publication was supported by the Deanship of Scientific Research at Prince Sattam bin Abdulaziz University, AlKharj, Saudi Arabia. The authors are also thankful to the Laboratory Director of Paediatric hospital, Riyadh, for giving us the specimens. 


\section{REFERENCES}

1. Billington, E., Phang, S.H., Gregson, D.B., Pitout, J.D.D., Ross, T., Church, D.L., Laupland, K.B., Parkins, M.D. 2014. Incidence, risk factors, and outcomes for Enterococcus spp. blood stream infections: a population-based study. Int J Infect Dis. 26, 76-82.

2. Billstrom, H., Sullivan, A., Lund, B. 2008. Crosstransmission of clinical Enterococcus faecium in relation to esp and antibiotic resistance. $J$ Appl Microbiol. 105, 21152122.

3. Garcia-G, M.C., Leo-Amador, G.E., Avila-Morales, J., Zaldivar-Lelo de Larrea, G., Sanchez-Corona, J. 2014. Vancomycin-resistant Enterococcus spp isolated from community acquired infections and colonizations in Queretaro City, Mexico. Am J Infect Control. 42, 577-580.

4. Quinones, D., Goni, P., Rubio, M.C., Duran, E., Gomez-Lus, R. 2005. Enterococci spp. isolated from Cuba: species frequency of occurrence and antimicrobial susceptibility profile. Diagn Microbiol Infect Dis. 51, 63-67.

5. Facklam, R.R., Carey, R. B. 1985. Streptococci and Aerococci, In Lennette E H, Balows A, Hausler WS, Shadomy Jr., HJ. (ed.), Manual of clinical microbiology. 4th ed. American Society for Microbiology, Washington, D.C. 154-175.

6. Musher, D.M. 1985. Streptococcus faecalis and other group D streptococci. In Mandell, G.L., Douglas, R.G., and Bennett, J.E. (ed.), Principles and practice of infectious diseases. 2nd ed. John Wiley \& Sons, Inc., New York. 11521155 .

7. Hidron, A.I., Edwards, J.R., Patel, J., Horan, T.C., Sievert, D.M., Pallock, D.A., Fridkin, S.K. 2008. NHSN Annual Update: Antimicrobial-Resistant Pathogens Associated With Healthcare-Associated Infections: Annual Summary of Data Reported to the National Healthcare Safety Network at the Centers for Disease Control and Prevention, 2006-2007. Infect Control Hosp Epidemiol. 29, 996-1011.

8. Fabretti, F., Huebner, J. 2005. Implant infections due to enterococci: role of capsular polysaccharides and biofilm. Int J Artif Organs. 28, 1079-1090.

9. Mathur, B., Agarwal, H.S., Maria, A. 2006. Acute renal failure in neonatal sepsis. Ind J Pedia. 73, 499-502.

10. Akortha, E., Ibadin, K. 2008. Incidence and antibiotic susceptibility pattern of Staphylococcus aureus among patients with urinary tract infection in UBTH Benin City, Nigeria. Afri J Biotech. 7, 1637-1640.

11. Owa, J.A. 1999. Urinary tract infections in children. In: Azubuike, J.C., Nkangineme, K.E., editors. Paediatrics and Child Health in a Tropical Region. Owerri: African Educational Services. pp. 480-481.

12. Shaikh, N., Morone, N. E., Bost, J.E., Farrell, M.H. 2008. Prevalence of urinary tract infection in childhood: a metaanalysis. Pedia Infect Dis J. 27, 302-308.
13. Runehagen, R., Kahlmeter, G. 2002. A 10- year study of the consumption of quinolones trimethoprim and mecillinam in relation to the development of antimicrobial resistance in a large number of species," in Proceeding of European Congress of Clinical Microbiology and Infectious Diseases.

14. Teixeira, L.M., Facklam, R.R. 2003 Enterococcus. In: Murray, P.R., Baron, E.J., Jorgensen, J.H., Pfaller, M.A., Yolken, R.H., editors. Manual of clinical microbiology. 8. ed. Washington: American Society for Microbiology. 422-433.

15. Hanson, L.A. 1976. Escherichia coli infections in childhood: significance of bacterial virulence and immune defence. Arch Dis Child. 51, 737-742.

16. Marild, S., Hansson, S., Jodal, U., Oden, A., Svedberg, K. 2004. Protective effect of breastfeeding against urinary tract infection. Acta Paediatr. 93, 164-168.

17. Hanson, L.A., Korotkova, M., Haversen, L., MattsbyBaltzer, I., Hahn-Zoric, M., Silfverdal, S.A., Strandvik, B., Telemo, E. 2002. Breast-feeding, a complex support system for the offspring. Pediatr Int. 44, 347-352.

18. Yamamoto, S., Tsukamoto, T., Terai, A., Kurazono, H., Takeda, Y., Yoshida, O. 1997. Genetic evidence supporting the fecal-perinealurethral hypothesis in cystitis caused by Escherichia coli. J Urol. 157, 1127-1129.

19. Clarridge, J.E., Pezzlo, M.T., Vosti, K.L., Weissfeld, A.S. 1987. Laboratory Diagnosis of Urinary Tract Infections. American Society for Microbiology, Washington DC.

20. Burnett, R.W., Haber, M.H., Hackel, E., Hanson, C.A., Keren, D.F., Lee-Lewandrowski, E. 1994. Clinical Laboratory Medicine, Williams \& Wilkins, Philadelphia. 1113-1120.

21. CLSI 2015. Performance standards for antimicrobial susceptibility testing: Twenty-fifth Informational Supplement M100-S25. Wayne, PA, USA.

22. Brion, C.P., Satlin, L.M., Edelmann, C.M., Macdonald, M.G.1994. Renal disease. In a very fletcher ma, eds. Neonatology: pathophysiology and management of the newborn. 4th ed. Philadelphia, Lippincott, 792-886.

23. Pollack, C.V. Jr., Pollack, E.S., Andrew, M.E. 1994. Suprapubic bladder aspiration versus urethral catheterization in ill infants: success, efficiency and complication rates. Ann Emerg Med. 23, 225-230.

24. Butler, K.M. 2006. Enterococcal infection in children. Semin Pediatr Infect Dis. 17, 128-139.

25. Paquette, K., Cheng, M.P., McGillivray, D., Lam, C., Quach, C. 2011. Is a lumbar puncture necessary when evaluating febrile infants (30 to 90 days of age) with an abnormal urinalysis? Pediatr Emerg Care. 27, 1057-1061.

26. Schnadower, D., Kuppermann, N., Macias, C.G., Freedman, S.B., Baskin, M.N., Ishimine, P., Scribner, C., Okada, P., Beach, H., Bulloch, B., Agrawal, D., Saunders, M., Sutherland, D.M., Blackstone, M.M., Sarnaik, A., McManemy, J., Brent, A., Bennett, J., Plymale, J.M., Solari, P., Mann, D.J., Dayan, P.S. 2010. Febrile infants with urinary tract infections at very low risk for adverse events and bacteremia. Pediatrics. 126, 1074-1083. 
27. Jerardi, K.E., Auger, K.A., Shah, S.S., Hall, M., Hain, P.D., Myers, A.1., Williams, D.J., Tieder, J.S. 2012. Discordant antibiotic therapy and length of stay in children hospitalized for urinary tract infection. J Hosp Med. 7, 622-627.

28. Phillips, J.R., Karlowicz, M.G. 1997. Prevalence of Candida species in hospital-acquired urinary tract infections in a neonatal intensive care unit. Pediatr Infect Dis J. 16, 190-194.

29. Ghosh, A., Poddar, S., Banerjee, S., Choudhury, J., Mukhopadhyay, M., Ray, J. 2018. Antibiotic Resistance in Community Acquired Urinary Tract Infection in Children: Data from a Tertiary Center in Eastern. India J Clin and Diagn Res. 12, 05-08.

30. James- Ellison, M.Y., Roberts, R., Verrier-jones, K., Williams, J.D., Topley, N. 1997. Mucosal immunity in the urinary tract: changes in $\operatorname{sIgA}$, FSC and total IgA with age and in urinary tract infection. Clin Nephrol. 48, 69-78.
31. Lo, D.S., Rodrigues, L., Koch, V.H.K., Gillo, A.E. 2018. Clinical and laboratory features of urinary tract infections in young infants. Braz J Nephrol. 40, 66-72.

32. Swaminathan, S., Alangaden, G.J. 2010. Treatment of Resistant Enterococcal Urinary Tract Infection. Curr Infect Dis Rep. 12, 455-464.

33. Coque, T.M., Singh, K.V., Weinstock, G.M., Murray, B.E. 1999. Characterization of dihydrofolate reductase genes from trimethoprim- susceptible and trimethoprim-resistant strains of Enterococcus faecalis. Antimicro Agents Chemother. 43, 141-147.

34. Gordon, S., Swenson, J.M., Hill, B.C., Pigott, N. E., Facklam, R.R., Cooksey, R.C., Thornsberry, C., Jarvis, W.R., Tenover, F.C. 1992. Antimicrobial susceptibility patterns of common and unusual species of enterococci causing infections in the United States. Enterococcal Study Group. $J$ Clin Microbiol. 30, 2373-2378. 